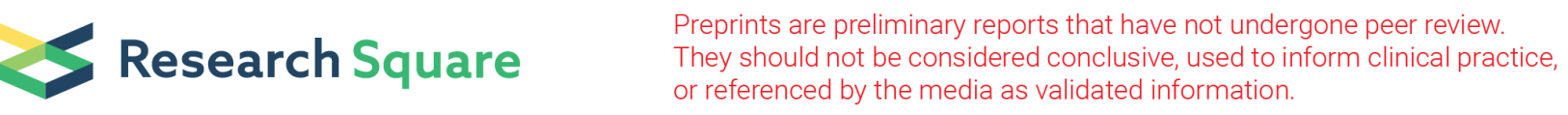

\title{
Insomnia and autonomic dysfunction may identify patients at risk for developing early Parkinson's disease dementia
}

\author{
Anamaria Jurcau ( $\sim$ anamaria.jurcau@gmail.com ) \\ Universitatea din Oradea https://orcid.org/0000-0002-0793-2877 \\ Vharoon Sharma Nunkoo \\ Spitalul Clinic Municipal Oradea
}

\section{Research article}

Keywords:

Posted Date: March 13th, 2019

DOI: https://doi.org/10.21203/rs.2.441/v1

License: (c) (i) This work is licensed under a Creative Commons Attribution 4.0 International License.

Read Full License 


\section{Abstract}

Background: The prospective study aims at identifying features predictive of early onset of dementia in patients with Parkinson's disease (PD). Methods: 89 non-demented PD patients underwent a complex evaluation (demographic data, UPDRS, Unified Multiple System Atrophy Rating Scale - UMSAR, Insomnia Severity Index - ISI, Neuro-Psychiatric Inventory - NPI, Hamilton Depression Rating Scale - HDRS, Mini Mental State Examination) at baseline and at 3-year follow-up. Results: At 3-year follow-up $43.8 \%$ of patients developed dementia. An ordinal regression of MMSE at follow-up showed that dementia developed in patients with autonomic dysfunctions (odds ratio $16.18,95 \% \mathrm{Cl} 3.16$ to $82.77, \mathrm{p}=0.001$ ), old age (odds ratio $1.24,95 \% \mathrm{Cl} 1.11$ to $1.39, \mathrm{p}<0.001$ ), and insomnia (odds ratio $1.23,95 \% \mathrm{Cl} 1.09$ to $1.38, p=0.001)$. Conclusion: Patients with signs of autonomic dysfunction and insomnia are at higher risk for developing dementia and deserve closer monitoring of cognitive symptoms.

\section{Background}

Neuropsychiatric disturbances accompany Parkinson's disease (PD) leading to a decline in the quality of life. PD patients frequently exhibit cognitive deficits as well, often evolving into dementia, causing increased caregiver burden, increased mortality, and often institutionalization. The prevalence of dementia in PD ranges between $24 \%$ and $50 \%$, and accounts for about $3 \%$ to $4 \%$ of all cases of dementia in the general population [1]. An incidence rate of approximately 100 per 1,000 patient-years has been estimated, more than 5 times that of age-matched controls [2].

Searching for the factors predictive of PD dementia is not only important in identifying high-risk patients for appropriate counseling and planning future care, but also in recognizing early signs of cognitive impairment for initiating therapeutic interventions. Advanced age is the most commonly established risk factor for dementia. Non-motor features such as REM sleep behavior disorder (RBD), baseline cognitive impairment, olfactory disturbances, and autonomic dysfunction have been identified as possible dementia predictors [3-6]. However, in many studies the tests were designed for clinical trial conditions, tests which are more difficult to apply as screening methods in real-life conditions and add to the costs of health care.

In our study, we assessed clinically, under conditions applicable in an outpatient care setting, a number of potential clinical risk factors for dementia in a 3-year prospective cohort study.

\section{Methods}

The present prospective study was conducted in accordance with the Declaration of Helsinki (1964) after approval of the Ethical Committee of the hospital.

\section{Patient selection}


Patients were recruited from the Neurology ward and outpatient clinic of our hospital. The period of enrollment extended from November 2012 to June 2014. A total of 130 patients were asked to undergo the neurological and psychological evaluation described below.

Exclusion criteria were:

- Secondary parkinsonisms or Pakinson-plus syndromes

- Dementia diagnosed at screening

- Significant comorbidities (e.g., cancer) which could impede the patient to complete the follow-up period

Parkinson's disease was diagnosed in accordance with the UK Brain Bank criteria [7]. Dementia was diagnosed by a certified psychiatrist according to the DSM-IV criteria taking into account also the particularities of Parkinson's disease dementia (impairment of at least two of the following domains: attention, executive function, visuo-constructive ability, memory) [8].

On the basis of the presence of dementia at enrollment 18 patients were excluded, and 9 denied enrollment for various reasons. Written consent to participate was obtained from all the 103 remaining patients, which were allowed to withdraw at any time. A number of 14 patients were lost to follow-up. Therefore we analyzed the data from 89 patients who completed the 3-year study period.

\section{Patient evaluation}

Patients underwent a complete neurological evaluation performed by a certified neurologist and a psychological evaluation at enrollment and after 3 years ( $36+/-3$ months) performed by a psychologist certified in clinical psychology. Meanwhile they had regular follow-up visits, dose adjustments of antiparkinsonian medication, and psychological and/or psychiatric evaluation as needed. Information on demographics, disease duration, medication was recorded. PD evaluation included:

\section{Motor variables:}

1.1 Unified Parkinson's Disease Rating Scale (UPDRS) - total and subscales I-IV [9].

1.2. Hoehn and Yahr staging [10].

1.3. records of falls and freezing in the history.

2. Autonomic variables - assessed through symptoms and signs of autonomic dysfunction (part III of the Unified Multiple System Atrophy Rating Scale) - at baseline [11].

3. Sleep disorders: by determining the Insomnia Severity Index [12]. Total score categories were considered as follows:

$0-7=$ No clinically significant insomnia 
$8-14=$ Subthreshold insomnia

15-21 = Clinical insomnia (moderate severity)

22-28 = Clinical insomnia (severe)

\section{Cognition:}

4.1. the MMSE score, using a cut point of 20 to differentiate between normal cognition or mild cognitive impairment vs. moderate/severe cognitive impairment [13].

\subsection{The clock-drawing test}

Patients with a MMSE score $\leq 20$, with executive dysfunctions outlined by the clock-drawing test, with visuo-constructive disabilities at the pentagon copying item of the MMSE, or patients with other symptoms suggestive of dementia were referred for psychiatric evaluation.

4.3. the 10-item version of the Neuropsychiatric Inventory (NPI) [14]. Screening questions for each of the 10 neuropsychiatric symptoms were posed first and if a positive response was obtained the symptom was further explored. Ratings from 1 through 4 were obtained for frequency and from 1 through 3 for severity of each symptom. A composite score for each symptom was calculated as the product of the frequency and severity (from 0 through 12) and was used for analysis.

5. Mood. The Hamilton Rating Scale for Depression, a 17 item questionnaire measuring psychological and physiological symptoms of depression [15] was administered to each patient at baseline, at 3-year follow-up visit, and between these 2 visits as needed. Severity ranges were considered as follows [16]: no depression (0-7); mild depression (8-16); moderate depression (17-23); and severe depression ( $\geq 24)$.

\section{Statistical analysis}

Statistical analysis was performed using IBM-SPSS version 19.0. Analyses consisted of description of the various scores, and calculation of proportions. Differences between groups were tested for statistical significance with the student's $t$ test. Factors associated with dementia were identified with a partial correlation analysis controlling for age and disease duration, after which they were included into an ordinal regression analysis. 95\% confidence intervals and odds-ratios were determined. Statistical significance was set at $\mathrm{p}<0.05$.

\section{Results}

The demographic and clinical description of the 89 patients included in the study is shown in table I. The gender distribution was $59.6 \%$ male and $40.4 \%$ female patients.

More than half of the patients $(53.9 \%)$ had complaints regarding their sleep pattern, while significant depressive clinical traits affected only $36 \%$ of patients at baseline. 26 patients $(29,2 \%)$ reported falls and 
freezing in their history.

After 3 years 57 patients (64\%) had clinically significant insomnia, while moderate-to-severe depression affected 47 patients (52.8\%). A number of 39 patients (43.8\%) were diagnosed with dementia according to the DSM IV criteria up to the 3-year follow-up.

Comparatively, the clinical and demographic description of patients who did develop dementia at 3-year follow-up versus those patients with preserved cognitive functions is provided in table II. All the analyzed parameters were significantly different between the 2 subgroups of patients, both at baseline and at follow-up, with $\mathrm{p}<0.001$ according to the independent samples t-test.

In order to see which clinical characteristics may predict a rapid evolution towards cognitive decline, we ran a partial correlation analysis between the dose of levodopa at baseline, the UPDRS score, the presence of autonomic dysfunctions (as indicated by 1 or more symptoms of autonomic dysfunction scored on the UMSAR scale), the Insomnia Severity Index, the score on the Hamilton Depression Rating Scale, the score of the Neuropsychiatric Inventory, and the MMSE at baseline (at the moment of inclusion into this study). Since with age and duration of disease the likelihood of developing dementia increases, we controlled for these 2 factors.

In patients who went on to developing dementia, the MMSE score at 3-year follow-up was significantly influenced only by the Insomnia Severity index (Pearson coefficient - $0.604, p<0.001$ ) and the presence of autonomic symptoms and signs (Pearson coefficient - 0.640, p < 0.001). Neither dose of levodopa, UPDRS score at baseline, nor Hamilton Depression Rating Scale score could predict which patients would develop dementia, while the correlation with the MMSE score at baseline failed to reach statistical significance $(p=0.065)$. However, patients more depressed were more likely to be on a higher dose of levodopa (Pearson coefficient 0.504, $p=0.001$ ).

In patients who did not develop dementia the MMSE score at 3-year follow-up was influenced most significantly again by the UMSAR score $(r=-0.396, p=0.005)$ and the ISI at baseline $(r=-0.431, p=$ 0.002 ), but also by the dose of levodopa (Pearson coefficient - 0.351, $p=0.014$ ), as well as by the severity of the disease, as indicated by the UPDRS score.

An ordinal regression analysis revealed further information. An increase in age (expressed in years) increases the odds of developing dementia with an odds ratio of 1.24 (95\% Cl, 1.11 to 1.39$)$, Wald $\chi 2=$ 12.496, $p<0.001$. Increased Insomnia Severity Indices led to increased odds of developing dementia with an odds ratio of $1.23(95 \% \mathrm{Cl}, 1.09$ to 1.38$)$, Wald $\chi 2=11.784, p=0.001$. The most striking was the relation to the autonomic dysfunctions presented by the patients at baseline. Having at least 2 signs of autonomic dysfunction increased the likelihood of developing dementia with an odds ratio of $16.18(95 \%$ $\mathrm{Cl}, 3.16$ to 82.77$)$, Wald $\chi 2=11.82, \mathrm{p}=0.001$.

\section{Discussion}


Of our 89 patients included in the study, 39 developed dementia in the 3 year follow-up period, at higher risk being those patients who presented signs of autonomic dysfunction and insomnia.

Almost half of the patients complained about their sleep patterns, while depression affected $37 \%$ of patients at inclusion in the study. After 3 years, sleep was disturbed in more than two third of patients, and depression rates exceeded $50 \%$. The figures provided for the depression rates by other studies resemble our findings $[17,18]$.

Focusing on the development of dementia, our study shows that a 3-year follow up was sufficient to show a significant decline in cognitive functions. It is true that the baseline MMSE was significantly lower in patients who were diagnosed with dementia after 3 years as compared with patients who were not demented after 36 months (21.39 vs. 26.38; $p<0.001)$. It may be that a simple MMSE test has too low specificity for evaluating cognition in Parkinson's disease since it focuses mainly on cortical functions while Parkinson's disease dementia is a subcortical type of dementia. It is possible that a part of these patients might have been diagnosed with dementia upon a more detailed testing. In addition, demented patients at follow up were older (69.67 years vs. 63.51 years; $p<0.001)$ and had a longer duration of the disease (7.62 vs. 5.59 years; $p<0.001$ ) when included in the study. However, even after controlling for these variables, the partial correlation analysis shows that the cognitive functions of patients diagnosed with dementia at follow-up were influenced by the UMSAR score and the Insomnia Severity Index at baseline.

The UMSAR score reflects signs of autonomic dysfunction. Previous cross-sectional studies have found strong correlations between cognition and orthostatic hypotension [19]. The cause for this strong relationship is unclear. It is possible that autonomic dysfunction is a marker of a more "diffuse" disease subtype, in which autonomic areas and cortical regions involved in dementia degenerate as well [20]. However, an alternative mechanism may also be discussed. In the general population low diastolic blood pressure has been linked to brain atrophy, particularly when associated with reduced cerebral blood flow [21]. Although cerebrovascular autoregulation mechanisms prevent orthostatic changes from reducing cerebral blood flow, moderate changes in PD which may associate multisystem and cardiovascular dysautonomia might cause significant cerebral hypoperfusion. Hypoxia and hypotension have been shown in animal models and human studies to cause neuronal damage and increases in accumulation of beta-amyloid and other pathologic proteins $[22,23]$. Therefore, one may reasonably hypothesize that repeated episodes of hypotension in PD over the years can augment damage to cortical neurons already made vulnerable by the synucleopathy. The findings of a prospective observational study showing that treatment of orthostatic hypotension improved cognition in dementia supports this hypothesis. [24].

Another predictor of dementia was the presence of insomnia. The sleep rhythm is driven by a central circadian pacemaker located in the suprachiasmatic nucleus of the anterior hypothalamus [25]. Studies of sleep deprivation and sleep restriction in healthy subjects revealed the importance of sleep in memory function and mental health. Experimentally induced sleep loss in healthy subjects is associated with impairments in a broad range of cognitive functions, and cognitive impairments have been identified in 
people with sleep disorders such as insomnia and sleep apnea. Sleep appears to be necessary for both memory encoding and consolidation. Sleep deprivation also affects emotional memory, with preservation of mainly negative compared to positive and neutral memories [25].

Disruptions in the sleep-wake cycle and sleep complaints are common complaints found in communitybased studies of older people. Over $50 \%$ of adults aged over 65 have at least one chronic sleep-related problem [26]. Advancing age with the associated decline in the melatonin and cortisol rhythms which entrain day-night activity patterns contribute to the changes in circadian rhythms which have been demonstrated in the elderly [27]. However, the link between sleep disturbances and Parkinson's disease is stronger. It has been estimated that a patient presenting typical Rapid Eye Movement sleep behavior disorder (REMBD) will have a 50\% chance of developing a parkinsonian syndrome within 5 years [28].

We did not find a significant correlation between the Hamilton Depression Rating Scale score and MMSE score, neither in patients converted to dementia nor in patients who did not develop dementia, although in the latter group of patients the link was stronger. Depression did not correlate with cognitive variables although it was more often present and more severe in patients with cognitive impairment. Previous studies have shown that mood changes in Parkinson's disease rather reflect disordered emotional functions [25]. It has been suggested that patients with no depression and no dementia have a normally functioning dopaminergic mesocortico-limbic system, while the opposite were true for PD patients with depression and/or cognitive impairment [25]. Supporting this hypothesis is the finding of a lower metabolic activity in the head of the caudate nucleus and in the inferior frontal cortex in the depressed PD patients as well as that of a significant inverse correlation between glucose metabolism in the inferior frontal cortex and depression scores [29].

The UPDRS score at baseline showed no significant correlation with the development of dementia or depression, highlighting the emphasis put by this rating scale on motor symptoms. Of a total of 179 possible points, 108 may be achieved from the motor items of the UPDRS scale.

An interesting finding is the link between dose of levodopa and decline of the MMSE at 3 year follow up. Levodopa is a precursor of dopamine. After crossing the blood-brain barrier it is decarboxylated to dopamine by aromatic amino acid decarboxylase present in neurons and glia, thereby contributing to its clinical efficacy in the treatment of Parkinson's disease. However, the presence of dopamine in the brain may be damaging as well. Un-sequestered dopamine is enzymatically degraded by monoamine oxidase $B$, a reaction during which hydrogen peroxide results as a by-product. Hydrogen peroxide is a powerful oxidant. In addition, auto-oxidation of dopamine results in highly reactive quinones which bind to proteins and interfere with the normal function of the latter. Increased oxidative stress also causes mitochondrial dysfunction and DNA damage, adding to the levodopa induced neurotoxicity [30]. A relatively recent study concluded that by a dysregulation of brain 5-hydroxytryptamine chronic levodopa treatment may contribute to non-motor symptoms related to spatial memory and fear [31]. The results presented provide further reasons for investigating adjunctive therapies to L-dopa for PD, such as antioxidants, as well as to delay treatment with levodopa for as long as possible, especially in young patients, although it is known 
that only mildly impaired PD patients may obtain symptom relief with anticholinergics or dopamine agonists [17].

We are aware of the limitations of our study, namely: the lack of recordings of family history of psychiatric disturbances, of personal history of head trauma, of non-parkinsonian medication use, the lack of detailed cognitive testing both at baseline and at follow up, using a complex battery of tests and use only of the MMSE and psychiatric evaluation, assessment of the presence of sleep disturbances only through the ISI, and a relatively small sample size, observed for a rather short period of time (36 months).

However, we desired to remain in the frame of an evaluation applicable as part of a usual outpatient visit. Those patients who have symptoms and/or signs which may indicate an early onset of dementia should undergo a more detailed testing and have visits scheduled more often.

\section{Conclusions}

In our opinion, our findings show that:

- Cognitive evaluation in initial and follow up assessment of Parkinson's disease patients may select those at risk for developing dementia raising the possibility of targeting coming neuroprotective agents at patients with this condition.

- Identifying and analyzing non-motor symptoms, especially symptoms of autonomic dysfunction and sleep disturbances, may identify patients at risk for PDD and enable correction of these factors as well as more frequent assessments to diagnose dementia at early stages.

It would be interesting to investigate whether correction of these dysfunctions would delay the development of dementia.

\section{Declarations}

\section{Ethics approval and consent to participate}

All patients gave written informed consent, and the ethical committee of the Clinical Municipal Hospital Oradea, Romania approved the study. Since patients with dementia at screening were not included in the study we did not ask for a family member or legal guardian's consent.

\section{Consent for publication}

Not applicable

\section{Availability of data and materials}

The datasets used during the current study are available from the corresponding author on reasonable request. 


\section{Competing interest}

The authors declare that they have no competing interests.

\section{Funding}

This research did not receive any specific grant from funding agencies in the public, commercial, or notfor-profit sectors.

\section{Authors' contribution}

Conceived and design the experiments: AJ, VSN . Data analysis: AJ. Manuscript preparation: AJ. Both authors read and approved the final manuscript.

\section{Acknowledgements}

The authors wish to thank psychologist Serac Popa Florina for her commitment and thorough psychological evaluation of the patients during the study.

\section{Abbreviations}

$\mathrm{Cl}$ - confidence interval; DSM IV - Diagnostic and Statistical Manual of Mental Disorders, 4th Edition; HDRS - Hamilton Depression Rating Scale; ISI - Insomnia Severity Index; MMSE - Mini Mental State Examination; NPI - Neuro-Psychiatric Inventory; PD - Parkinson's Disease; RBD - REM sleep behavior disorders; REM - Rapid eye movement; UMSAR - Unified Multiple System Atrophy Rating Scale; UPDRS - Unified Parkinson's Disease Rating Scale

\section{Tables}

Due to technical limitations, Tables 1 and 2 are only available as a download in the supplemental files section.

\section{References}

1. Aarsland D, Zaccai J, Brayne C. A systematic review of prevalence of dementia in Parkinson's disease. Mov Disord 2005;20:1255-63

2. Hobson P, Meara J. Risk and incidence of dementia in a cohort of older subjects with Parkinson's disease in the United Kingdom. Mov Disord 2004;19:1043-9.

3. Postuma RB, Bertrand JA, Montplaisir J, et al. Rapid eye movement sleep behavior disorder and risk of dementia in Parkinson's disease: a prospective study. Mov Disord 2012;27:720-6. 
4. Williams-Gray $\mathrm{CH}$, Foltynie T, Brayne $\mathrm{CEG}$, et al. Evolution of cognitive dysfunction in an incident Parkinson's disease cohort. Brain 2007;130:1787-98.

5. Baba T, Kikuchi A, Hirayama K, et al. Severe olfactory dysfunction is a prodromal symptom of dementia associated with Parkinson's disease: a 3 year longitudinal study. Brain 2012;135:161-9.

6. Fereshtehnejad S, Romenets SR, Anang JBM, Latreille V, Gagnon J, Postuma RB. New clinical subtypes of Parkinson Disease and their longitudinal progression. A prospective cohort comparison with other phenotypes. JAMA Neurol. 2015;72:863-73.

7. Hughes AJ, Daniel SE, Kilford L, Lees AJ. Accuracy of clinical diagnosis of idiopathic Parkinson's disease. A clinico-pathological study of 100 cases. J Neurol Neurosurg Psychiatry 1992;55:181-4.

8. Poewe W, Gauthier S, Aarsland D, Leverenz JB, Barone P, Weintraub D, et al. Diagnosis and management of Parkinson's disease dementia. Int J Clin Pract 2008; 62:1581-1587

9. Goetz CG, et al. Movement Disorder Society-sponsored revision of the Unified Parkinson's Disease Rating Scale (MDS-UPDRS): scale presentation and clinimetric testing results. Mov Disord 2008;23:2129-70.

10. Goetz CG, Poewe W, Rascol O, Sampaio C, et al. Movement Disorder Society Task Force report on the Hoehn and Yahr staging scale: status and recommendations. Mov Disord 2004;19:1020-8.

11. Wenning GK, Tison F, Seppi K, et al. Development and validation of the Unified Multiple System Atrophy Rating Scale (UMSARS). Mov Disord 2004;19:1391-1402.

12. Bastien $\mathrm{CH}$, Vallieres A, C. M. Morin CM. Validation of the Insomnia Severity Index as an outcome measure for insomnia research. Sleep Med 2001;2: 297-307.

13. Folstein MF, Folstein SE, McHugh PR: "Mini-mental state: A practical method for grading the cognitive state of patients for the clinician." J Psychiatr Res 1975;12:189-98.

14. Neuropsychiatric Inventory. www.npitest.net/about-npi.html, accessed April 19, 2011.

15. Hamilton Depression Rating Scale. www.psy-world.com/online_hamd.htm, accessed March 21, 2011

16. Zimmermann M, Martinez JH, Young D, Dalrymple K. Severity classification on the Hamilton depression rating Scale. J Affect Disord 2013;150:384-8.

17. Girotti F, Soliveri P, Carella F, et al. Dementia and cognitive impairment in Parkinson's disease. J Neurol Neurosurg Psychiatry 1988;51:1498-1502.

18. Starkstein SE, Mayberg HS, Leiguarda R, Preziosi TJ, Robinson RG. A prospective longitudinal study of depression, cognitive decline, and physical impairments in patients with Parkinson's disease. J Neurol Neurosurg Psychiatry 1992;55:377-82. 
19. Pilleri M, Facchini S, Gasparoli E, et al. Cognitive and MRI correlates of orthostatic hypotension in Parkinson's disease. J Neurol 2013;260:253-9.

20. Anang JBM, Gagnon JF, Bertrand JA, et al. Predictors of dementia in Parkinson disease. A prospective cohort study. Neurology 2014;83:1253-60.

21. Frewen J, Finucane C, Sawa GM, Boyle G, Kenny RA. Orthostatic hypotension is associated with lower cognitive performance in adults aged 50 plus with supine hypertension. J Gerontol A Biol Sci Med Sci 2013;69:878-85.

22. Liu H, Xing A, Wang X, Liu G, Li L. Regulation of beta-amyloid level in the brain of rats with cerebrovascular hypoperfusion. Neurobiol Aging 2012;33:826.e31-42.

23. de la Torre JC. Cardiovascular risk factors promote brain hypoperfusion leading to cognitive decline and dementia. Cardiovasc Psychiatry Neurol 2012;2012:367516.

24. Freidenberg DL, Shaffer LE, Macalester S, Fannin EA. Orthostatic hypotension in patients with dementia: clinical features and response to treatment. Cogn Behav Neurol 2013;26:105-20.

25. Anderson KN, Bradley AJ. Sleep disturbance in mental health problems and neurodegenerative disease. Nat Sci Sleep 2013;31:61-75.

26. Foley DJ, Monjan AA, Brown SL, Simonsick EM, Wallace RB, Blazer DG. Sleep complaints among elderly persons: an epidemiologic study of three communities. Sleep 1995;16:425-33.

27. Mazzoccoli G, Vendemiale G, La Viola M, et al. Circadian variations of cortisol, melatonin and lymphocyte subpopulations in geriatric age. Int J Immunopathol Pharmacol 2010;23:289-96.

28. Fulda S. Idiopathic REM sleep behavior disorder as a long-term predictor of neurodegenerative disorders. EPMA 2011;2:451-8.

29. Mayberg HS, Starkstein SE, Sadzot B, et al. Selective inferior frontal lobe hypometabolism in depressed patients with Parkinson's disease. Ann. Neurol 1990;28:57-64.

30. Stansley BJ, Yamamoto BK. L-dopa-induced dopamine synthesis and oxidative stress in serotoninergic cells. Neuropharmacology 2013;67:243-51.

31. Stansley BJ, Yamamoto BK. Behavioral impairments and serotonin reductions in rats after chronic Ldopa. Psychopharmacology (Berl) 2015;232:3203-13.

\section{Supplementary Files}

This is a list of supplementary files associated with this preprint. Click to download. 
- supplement1.pdf

- supplement2.pdf 